\title{
Generalized Convolution Spectral Mixture for Multi-task Gaussian Processes
}

\author{
Kai Chen \\ Shenzhen Institutes of Advanced Technology, Chinese Academy of Sciences \\ Shenzhen College of Advanced Technology, University of Chinese Academy of Sciences \\ Institute for Computing and Information Sciences, Radboud University \\ kchen@cs.ru.nl \\ Twan van Laarhoven \\ Institute of Management, Science and Technology, Open University of the Netherlands \\ Institute for Computing and Information Sciences, Radboud University \\ mail@twanvl.nl \\ Perry Groot \\ Institute for Computing and Information Sciences, Radboud University \\ perry.groot@science.ru.nl \\ Jinsong Chen \\ Shenzhen Institutes of Advanced Technology, Chinese Academy of Sciences \\ js.chen@siat.ac.cn \\ Elena Marchiori \\ Institute for Computing and Information Sciences, Radboud University \\ elenam@cs.ru.nl
}

\begin{abstract}
Multi-task Gaussian processes (MTGPs) are a powerful approach for modeling structured dependencies among multiple tasks. Researchers on MTGPs have contributed to enhance this approach in various ways. Current MTGP methods, however, cannot model nonlinear task correlations in a general way. In this paper we address this problem. We focus on spectral mixture (SM) based kernels and propose an enhancement of this type of kernels, called multi-task generalized convolution spectral mixture (MT-GCSM) kernel. The MT-GCSM kernel can model nonlinear task correlations and mixtures dependency, including time and phase delay, not only between different tasks but also within a task at the spectral mixture level. Each task in MT-GCSM has its own generalized convolution spectral mixture kernel (GCSM) with a different number of convolution structures and all spectral mixtures from different tasks are dependent. Furthermore, the proposed kernel uses inner and outer full cross convolution between base spectral mixtures, so that the base spectral mixtures in the tasks are not necessarily aligned. Extensive experiments on synthetic and real-life datasets illustrate the difference between MT-GCSM and other kernels as well as the practical effectiveness of MT-GCSM.
\end{abstract}




\section{Introduction}

Gaussian Processes (GPs) are an elegant Bayesian approach to model an unknown function. They provide regression models where a posterior distribution over the unknown function is maintained as evidence is accumulated. This allows Gaussian processes to learn complex functions if a large amount of evidence is available and makes them robust against overfitting in the presence of little evidence [1, 2]. A GP can model a large class of phenomena through the choice of its kernel which characterizes one's assumption on how the unknown function autocovaries. The choice of kernel, however, is a core aspect of the GP design, since the posterior distribution can significantly vary for different kernels. As a consequence, various kernels (e.g., Squared Exponential, Periodic, and Matérn) and kernel design methods have been proposed [2]. The extension of GPs to multiple sources of data is known as multi-task Gaussian processes (MTGPs). MTGPs model temporal or spatial relationships among infinitely many random variables, as scalar GPs, but also account for the statistical dependence across different sources of data (or tasks) [3, 4, [1, 6, , , 8]. How to choose an appropriate kernel to jointly model the cross covariance between tasks and auto-covariance within each task is the core aspect of MTGPs design [9, [10, 11, 4, 12, 13]].

Early approaches to MTGPs, like the Linear Model of Coregionalization (LMC [14, 3, 7] focused on linear combinations of independent single-output GPs. More expressive methods like the multi-kernel method [11] and the convolved latent function framework [15, 16, 17, 18] consider convolution to construct cross-covariance functions, and assume that each task has its own kernel. The use of spectral mixture (SM) kernels has further boosted the development of MTGP methods. Specifically, the expressiveness power of MTGP methods with SM kernels has increased during the past years: first the SM-LMC kernel was proposed [19, 20], which just uses independent spectral mixtures and global linear weights; then the Cross-Spectral Mixture (CSM) kernel [21], a more flexible kernel which considers the power and phase correlation between multiple tasks. CSM cannot capture complicated cross correlations because it only considers phase dependencies between tasks. Therefore the Multi-Output Spectral Mixture kernel (MOSM) was proposed [22] which addresses this limitation. MOSM, however, considers task level correlations within each spectral mixture by using independent components. MOSM has three limitations: all tasks have the same number of components, components in different tasks should be aligned, and spectral mixture level dependency within each task is ignored. Recently the generalized convolution spectral mixture of coupling coregionalization (GCSM-CC) kernel [23] explicitly extended previous works to model nonlinear correlations between tasks and dependencies between spectral mixtures and introduced coupling coregionalization to learn task level correlations. This means GCSM-CC only addresses the last mentioned limitation of MOSM, but that, as a result of using coupling coregionalization [23], tasks in GCSM-CC share the same kernel, and hyper-parameters in coregionalization terms involving task correlations are global and linear.

In this paper we fully address structure modeling of task level correlations and spectral level dependencies in MTGP and propose the multi-task generalized convolution spectral mixture (MT-GCSM) kernel. In MT-GCSM, we consider a two-level type of dependency: at the task level and at the spectral mixture level using generalized convolution spectral mixture kernel [24]. Without the restriction that all tasks should have the same number of components, both task level correlation and spectral mixture level dependency are fully convolved with time and phase delay. In the proposed kernel, each task has its own Generalized Convolution Spectral Mixture (GCSM) kernel [24] characterized by the task structure. Notably, without increasing the hyper-parameter space, MT-GCSM can involve hundreds of convolution structures. When only a single task is available, MT-GCSM reduces to the GCSM which has been shown to be a generalization of the ordinary SM kernel.

Specifically, we address the following questions. (1) How to model spectral mixture level dependency within a task? (2) How to build generalized spectral mixtures between tasks for modeling task level correlation? (3) How to incorporate inner spectral mixture level dependency and cross task level correlation simultaneously? (4) What's the relationship between MT-GCSM and other SM-based kernels?

The remainder of this paper is organized as follows. Background on GPs and SM kernels is given in Section [2. Our MT-GCSM kernel is described in Section B]. Section \# compares MT-GCSM with SM-based kernels for MTGP in terms of hyper-parameter space and degrees of freedom. Section [5] illustrates the beneficial aspects of MT-GCSM. Section 6 describes results of experiments on synthe- 
tic and real-world datasets. A summary of the paper's contributions, concluding remarks, and future work on this topic are given in the Section $\square$.

\section{Backgroud}

We start with some background information on GPs, multi-task GPs, and spectral mixture kernels.

\subsection{Gaussian processes}

A Gaussian process defines a distribution over functions, specified by its mean and covariance function [2]. The mean function $m(x)$ and covariance function $k\left(x, x^{\top}\right)$ can be written as

$$
\begin{aligned}
m(x) & =\mathbb{E}[f(x)] \\
k\left(x, x^{\top}\right) & =\mathbb{E}\left[(f(x)-m(x))\left(f\left(x^{\top}\right)-m\left(x^{\top}\right)\right)\right]
\end{aligned}
$$

where $x$ is an arbitrary input variable in $\mathbb{R}^{P}$. The covariance function $k$ mapping two random variables into $\mathbb{R}^{P}$, is applied to construct a positive definite covariance matrix, here denoted by $K$. Given $m(x)$ and $k(x)$, we can define a GP as

$$
f(x) \sim \mathcal{G} \mathcal{P}\left(m(x), k\left(x, x^{\top}\right)\right)
$$

Without loss of generality we assume the mean of a GP to be zero. By placing a GP prior over functions through the choice of kernels and parameter initialization, and the training data, we can predict the unknown value $\bar{y}_{*}$ and its variance $\mathbb{V}\left[y_{*}\right]$ (that is, its uncertainty) for a test point $x_{*}$ using the following key predictive equations for GP regression [2]:

$$
\begin{aligned}
\bar{y}_{*} & =K^{\top}\left(\mathbf{x}, x_{*}\right)\left(K\left(\mathbf{x}, \mathbf{x}^{\top}\right)+\sigma_{n}^{2} I\right)^{-1} \mathbf{y} \\
\mathbb{V}\left[y_{*}\right] & =k\left(x_{*}, x_{*}\right)-K^{\top}\left(\mathbf{x}, x_{*}\right)\left(K\left(\mathbf{x}, \mathbf{x}^{\top}\right)+\sigma_{n}^{2} I\right)^{-1} K\left(\mathbf{x}, x_{*}\right)
\end{aligned}
$$

where $\mathbf{x}$ is an input vector and $\mathbf{y}$ is the observed value corresponding to input $\mathbf{x}$. Typically, GPs contain free parameters, called hyper-parameters, which can be optimized by minimizing the Negative Log Marginal Likelihood (NLML). The NLML is defined as follows:

$$
\begin{aligned}
\mathrm{NLML} & =-\log p(\mathbf{y} \mid \mathbf{x}, \Theta) \\
& \propto \overbrace{\frac{1}{2} \mathbf{y}^{\top}\left(K+\sigma_{n}^{2} I\right)^{-1} \mathbf{y}}^{\text {model fit }}+\overbrace{\frac{1}{2} \log \left|K+\sigma_{n}^{2} I\right|}^{\text {complexity penalty }}
\end{aligned}
$$

where $K=K\left(\mathbf{x}, \mathbf{x}^{\top}\right), \Theta$ are the hyper-parameters of the kernel function, and $\sigma_{n}^{2}$ is the noise level. The NLML above directly follows from the observation that $\mathbf{y} \sim N\left(0, K+\sigma_{n}^{2} I\right)$.

In multi-task GP (MTGP), we have multiple sources of data which specify related tasks. The construction of the MTGP covariance function $k_{\mathrm{MTGP}}$ models dependencies between pairs of points from two tasks.

\subsection{Spectral mixture kernel}

Usually, the smoothness and generalization properties of GPs depend on the kernel function and its hyper-parameters $\Theta$. Choosing an appropriate kernel function and its initial hyper-parameters based on prior knowledge from the data are the core steps of a GP. Various kernel functions have been proposed [2], such as Squared Exponential (SE), Periodic (PER), and general Matérn (MA). Recently new covariance kernels have been proposed in [19, 25], called Spectral Mixture (SM) kernels. A SM kernel, here denoted by $K_{\mathrm{SM}}$, is derived through modeling a spectral density (Fourier transform of a kernel) with Gaussian mixtures. A desirable property of SM kernels is that they can be used to reconstruct other popular standard covariance kernels. According to Bochner's Theorem [26], the properties of a stationary kernel entirely depend on its spectral density. With enough components $k_{\mathrm{SM}}$ can approximate any stationary covariance kernel [25].

$$
k_{\mathrm{SM}}(\tau)=\sum_{q=1}^{Q} w_{q} \cos \left(2 \pi \boldsymbol{\mu}_{q} \tau^{\top}\right) \prod_{p=1}^{P} \exp \left(-2 \pi^{2} \tau^{2} \Sigma_{q}^{(p)}\right)
$$


where $Q$ is the number of components, $P$ is the dimension of dataset, $w_{q}, \boldsymbol{\mu}_{q}=\left[\mu_{q}^{m}, \ldots, \mu_{q}^{(P)}\right]$, and $\Sigma_{q}=\operatorname{diag}\left(\left[\left(\sigma_{q}^{2}\right)^{m}, \ldots,\left(\sigma_{q}^{2}\right)^{(P)}\right]\right)$ are weight, mean, and variance of the $q$-th mixture component in frequency domain, respectively. The variance $\sigma_{q}^{2}$ can be thought of as an inverse length-scale, $\mu_{q}$ as a frequency, and $w_{q}$ as a contribution.

Bochner's Theorem [26, 27] indicates a direction on how to construct a valid kernel from the frequency domain. This implies that this kind of kernels can also be transformed between time domain and frequency domain. Using the following definition, the spectral density of kernel function $k(\tau)$ can be given by its Fourier transform:

$$
\hat{k}(s)=\int_{-\infty}^{\infty} k(\tau) e^{-2 \pi i \tau s} d \tau
$$

Furthermore, the inverse Fourier transform of spectral density $\hat{k}(s)$ is the original kernel function $k(\tau)$.

$$
k(\tau)=\int_{-\infty}^{\infty} \hat{k}(s) e^{2 \pi i \tau s} d s
$$

We will use a hat $\hat{k}(s)$ to denote the spectral density of a covariance function $k$ in the frequency domain. From Bochner's theorem [26, 27] $k(\tau)$ and $\hat{k}(s)$ are Fourier duals of each other. For SM kernel [19], using Fourier transform of the spectral density $\hat{k}_{\mathrm{SM}}(\mathbf{s})=\left[\varphi_{\mathrm{SM}}(\mathbf{s})+\varphi_{\mathrm{SM}}(-\mathbf{s})\right] / 2$ where $\varphi_{\mathrm{SM}}(\mathbf{s})=\mathcal{N}(\mathbf{s} ; \boldsymbol{\mu}, \Sigma)$ is a symmetrized scale-location mixture of Gaussians in the frequency domain, we have

$$
\begin{aligned}
k_{\mathrm{SM}}(\tau) & =\mathcal{F}_{s \rightarrow \tau}^{-1}\left[\sum_{q=1}^{Q} w_{q} \hat{k}_{\mathrm{SM}}(\mathbf{s})\right](\tau) \\
& =\sum_{q=1}^{Q} w_{q} \mathcal{F}_{s \rightarrow \tau}^{-1}\left[\left(\varphi_{\mathrm{SM}}(\mathbf{s})+\varphi_{\mathrm{SM}}(-\mathbf{s})\right) / 2\right](\tau)
\end{aligned}
$$

\section{Multi-task generalized convolution SM kernels}

We now address the first three questions mentioned in Section $\square$.

\subsection{Generalized convolution spectral mixture kernel within task}

In [24] we used convolution to model spectral mixture level dependency with a quadratic number of convolution structures in a single task. The resulting GCSM kernel can be formalized as follow:

$$
\begin{aligned}
k_{\mathrm{GCSM}}(\tau)= & \mathcal{F}_{s \rightarrow \tau}^{-1}\left[\left(\sum_{i=1}^{Q} \sqrt{w_{i} \varphi_{\mathrm{GCSM} i}(\mathbf{s})}\right) \cdot\left(\sum_{j=1}^{Q} \sqrt{w_{j} \overline{\varphi_{\mathrm{GCSM}}(\mathbf{s})}}\right)\right. \\
& +\left(\sum_{i=1}^{Q} \sqrt{w_{i} \varphi_{\mathrm{GCSM} i}(-\mathbf{s})}\right) \cdot\left(\sum_{j=1}^{Q} \sqrt{\left.\left.w_{j} \overline{\varphi_{\mathrm{GCSM} j}(-\mathbf{s})}\right)\right] / 2}\right. \\
= & \sum_{i=1}^{Q} \sum_{j=1}^{Q} \sqrt{w_{i} w_{j}}\left|\frac{\sqrt{4 \Sigma_{i} \Sigma_{j}}}{\Sigma_{i}+\Sigma_{j}}\right|^{\frac{1}{2}} \exp \left(-\frac{1}{4}\left(\boldsymbol{\mu}_{i}-\boldsymbol{\mu}_{j}\right)^{\top}\left(\Sigma_{i}+\Sigma_{j}\right)^{-1}\left(\boldsymbol{\mu}_{i}-\boldsymbol{\mu}_{j}\right)\right) \\
& \times \exp \left(-\pi^{2} \frac{\left(2 \tau-\left(\boldsymbol{\theta}_{i}-\boldsymbol{\theta}_{j}\right)\right)^{\top} \Sigma_{i} \Sigma_{j}\left(2 \tau-\left(\boldsymbol{\theta}_{i}-\boldsymbol{\theta}_{j}\right)\right)}{\Sigma_{i}+\Sigma_{j}}\right) \\
& \times \cos \left(\pi\left(\frac{\left(2 \tau-\left(\boldsymbol{\theta}_{i}-\boldsymbol{\theta}_{j}\right)\right)^{\top}\left(\Sigma_{i} \boldsymbol{\mu}_{j}+\Sigma_{j} \boldsymbol{\mu}_{i}\right)}{\Sigma_{i}+\Sigma_{j}}-\left(\boldsymbol{\phi}_{i}-\boldsymbol{\phi}_{j}\right)\right)\right)
\end{aligned}
$$

where $Q$ is the number of auto-convolution spectral mixtures in the GCSM kernel, $\varphi_{\mathrm{GCSM} i}(\mathbf{s})=$ $\mathcal{N}\left(\mathbf{s} ; \boldsymbol{\mu}_{i}, \Sigma_{i}, \boldsymbol{\theta}_{i}, \boldsymbol{\phi}_{i}\right)$ is a spectral density of GCSM in the frequency domain and the overline symbol 
denotes the complex conjugate operator.

$$
\varphi_{\mathrm{GCSM} i}(\mathbf{s})=\frac{1}{\sqrt{(2 \pi)^{P}\left|\Sigma_{i}\right|}} \exp \left(-\frac{\left(\mathbf{s}-\boldsymbol{\mu}_{i}\right)^{\top}\left(\mathbf{s}-\boldsymbol{\mu}_{i}\right)}{2 \Sigma_{i}}\right) \exp \left(-2 \pi \boldsymbol{\theta}_{i} \mathbf{s} i-2 \pi \phi_{i} i\right)
$$

where $\boldsymbol{\theta}$ and $\phi$ are time delay and phase delay. Using GCSM we can model the dependency related to time and phase delay between spectral mixtures in a single task (see Figure $\mathbb{W}$ ). The single task GCSM can be seen as an inner full cross convolution spectral mixture in multi-task GCSM kernel.

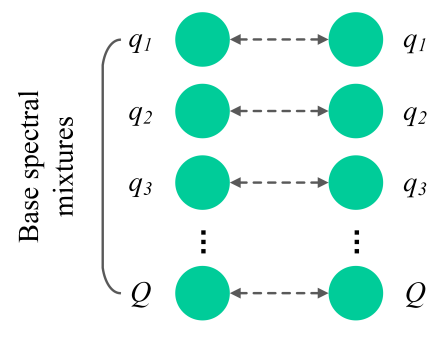

(a) Ordinary SM

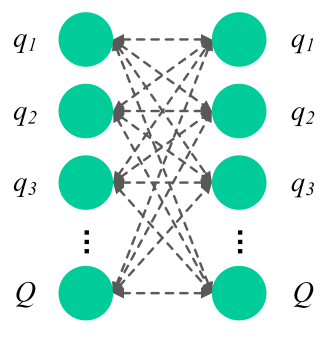

(b) GCSM

Figure 1: GCSM and SM with the same number of $Q$ base spectral mixtures within a task. (a) the auto-convolution of base spectral mixtures in SM. (b) the cross and auto-convolution between base spectral mixtures in GCSM. Each circle represents a base spectral mixtures in GCSM. All circles have the same green color because they come from the same task.

\subsection{Outer cross convolution spectral mixtures between tasks}

Here we extend GCSM to a multi-task scenario. While in single task GP time and phase delay dependency exist within the task, in MTGP time and phase delay dependencies exist between different spectral mixtures from different tasks. Similar to GCSM in single task GPs, by considering distributivity of convolution, we construct full cross convolution between, say, task $T_{m}$ and task $T_{m^{\prime}}$. Here tasks $T_{m}$ and $T_{m^{\prime}}$ have different number of base spectral mixtures $Q^{(m)}$ and $Q^{\left(m^{\prime}\right)}$, respectively. Combining the inner full cross convolution of spectral mixtures within task (single task GCSM) and the outer full cross convolution spectral mixture between tasks, we can construct the so-called multi-task generalized convolution spectral mixture kernel (MT-GCSM), capable to model full dependency structure for MTGP at spectral mixture level. Here, time and phase delay dependencies are represented not only inside each task but also across tasks. The convolution relation is shown in Figure 2. The form of MT-GCSM between two tasks $T_{m}$ and $T_{m^{\prime}}$ is

$$
\begin{aligned}
k_{\mathrm{MT}-\mathrm{GCSM}}^{\mathrm{T}_{m} \times \mathrm{T}_{m^{\prime}}}(\tau)= & \mathcal{F}_{s \rightarrow \tau}^{-1}\left[\left(\sum_{i=1}^{Q^{(m)}} \sqrt{w_{i}^{m} \varphi_{\mathrm{GCSM} i}^{m}(\mathbf{s})}\right) \cdot\left(\sum_{j=1}^{Q^{\left(m^{\prime}\right)}} \sqrt{w_{j}^{m^{\prime}} \overline{\varphi_{\mathrm{GCSM} j}^{m^{\prime}}(\mathbf{s})}}\right)\right. \\
& \left.+\left(\sum_{i=1}^{Q^{(m)}} \sqrt{w_{i}^{m} \varphi_{\mathrm{GCSM} i}^{m}(-\mathbf{s})}\right) \cdot\left(\sum_{j=1}^{Q^{\left(m^{\prime}\right)}} \sqrt{w_{j}^{m^{\prime}} \overline{\varphi_{\mathrm{GCSM} j}^{m^{\prime}}(-\mathbf{s})}}\right)\right] / 2 \\
= & \sum_{i=1}^{Q^{(m)}} \sum_{j=1}^{Q^{\left(m^{\prime}\right)}} \sqrt{w_{i}^{m} w_{j}^{m^{\prime}}}\left|\frac{\sqrt{4 \Sigma_{i}^{m} \Sigma_{j}^{m^{\prime}}}}{\sum_{i}^{\frac{1}{2}}+\Sigma_{j}^{m^{\prime}}}\right|^{\frac{1}{2}} \\
& \times \exp \left(-\frac{1}{4}\left(\boldsymbol{\mu}_{i}^{m}-\boldsymbol{\mu}_{j}^{m^{\prime}}\right)^{\top}\left(\Sigma_{i}^{m}+\Sigma_{j}^{m^{\prime}}\right)^{-1}\left(\boldsymbol{\mu}_{i}^{m}-\boldsymbol{\mu}_{j}^{m^{\prime}}\right)\right) \\
& \times \exp \left(-\pi^{2} \frac{\left(2 \tau-\left(\boldsymbol{\theta}_{i}^{m}-\boldsymbol{\theta}_{j}^{m^{\prime}}\right)\right)^{\top} \Sigma_{i}^{m} \Sigma_{j}^{m^{\prime}}\left(2 \tau-\left(\boldsymbol{\theta}_{i}^{m}-\boldsymbol{\theta}_{j}^{m^{\prime}}\right)\right)}{\Sigma_{i}^{m}+\Sigma_{j}^{m^{\prime}}}\right) \\
& \times \cos \left(\pi\left(\frac{\left(2 \tau-\left(\boldsymbol{\theta}_{i}^{m}-\boldsymbol{\theta}_{j}^{m^{\prime}}\right)\right)^{\top}\left(\sum_{i}^{m} \boldsymbol{\mu}_{j}^{m^{\prime}}+\Sigma_{j}^{m^{\prime}} \boldsymbol{\mu}_{i}^{m}\right)}{\Sigma_{i}^{m}+\Sigma_{j}^{m^{\prime}}}-\left(\boldsymbol{\phi}_{i}^{m}-\boldsymbol{\phi}_{j}^{m^{\prime}}\right)\right)\right)
\end{aligned}
$$


where $Q^{(m)}$ and $Q^{\left(m^{\prime}\right)}$ are the number of base spectral mixtures in tasks $T_{m}$ and $T_{m^{\prime}}$, respectively. In our setting, GCSM kernels in different tasks can have different number of spectral mixtures, depending on the task's complexity. By analyzing the spectral density of a task in the frequency domain one can gain insight into its complexity. Note that existing SM-based kernels, for instance SM-LMC, MOSM and CSM, assume that all tasks should have the same number of spectral mixtures and that spectral mixtures should be aligned. Our kernel does not have such constraints.

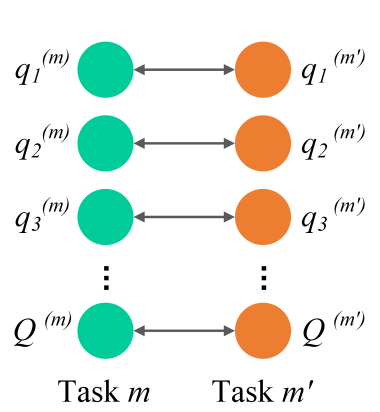

(a) MOSM

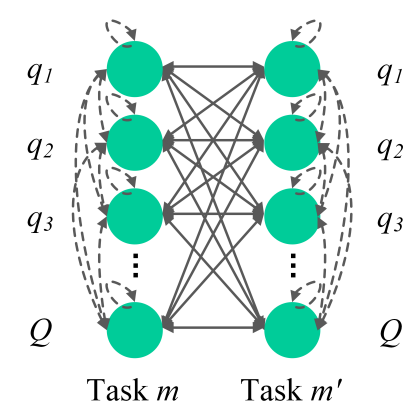

(b) GCSM-CC

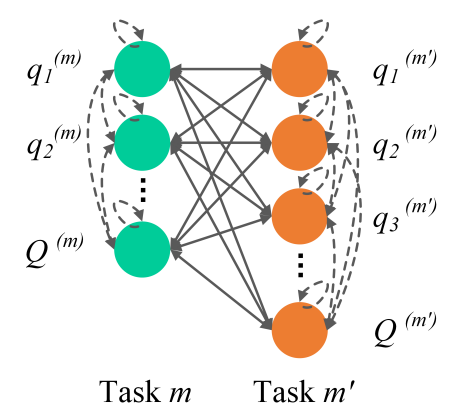

(c) MT-GCSM

Figure 2: Convolution relation in MT-GCSM. (a) mixture-wised dependencies in MOSM and $Q^{(m)}=Q^{\left(m^{\prime}\right)}$. (b) spectral mixture level dependent and task level coupled GCSM-CC with using the same GCSM kernel for all tasks. (c) MT-GCSM with $Q^{(m)} \neq Q^{\left(m^{\prime}\right)}$.

The proposed MT-GCSM kernel is illustrated in Figure D. Each connection in MT-GCSM represents a convolution structure. While, for GCSM-CC and MT-GCSM, the dashed line in Figure $\square$ denotes the inner cross convolution structures. The solid lines in CGSM-CC and MT-GCSM are coupling coregionalization terms and outer cross convolution structures, respectively. Note that in GCSM-CC, tasks $T_{m}$ and $T_{m^{\prime}}$ share the same GCSM kernel, so the circles with the same green color.

Tasks in MT-GCSM have different number of components. When task 1 is equal to task 2 , there is no cross spectral mixture level convolution between tasks and the MT-GCSM reduces into GCSM. Furthermore, without considering spectral mixture dependencies, GCSM reduces into ordinary SM. All dashed lines in MT-GCSM can be seen as a set of inner component dependency. All solid lines in MT-GCSM can be seen as a set of cross tasks correlation. Both dashed lines and solid lines have the same convolution scale because both them based on convolution of base spectral mixtures.

In MT-GCSM, arbitrary MTGP kernel $\Omega$ constructed by MT-GCSM with arbitrary number of tasks fulfills the positive semi-definite condition $(\Omega \geq 0$, the detailed proof is given in the appendix). The proof of positive semi-definite condition of MT-GCSM is different from that for GCSM because of the introduction of outer full cross convolution terms between tasks.

\section{Relation to other kernels}

Here we address the last question mentioned in Section $\mathrm{l}$. A lot of improvements and applications related to MTGPs have been achieved in previous works, like [3, 7, 11, 15, 20, 21, 22]. Since the introduction of SM kernels [25, 28], MTGPs with SM kernels [25, 28, 29, 30, 31, 32, 33] showed a strong learning ability and interpretation. Here we focus on MTGP methods based on such kernels [20, 21, 22]. The first MTGP using a SM kernel is based on the LMC framework [20] to construct a Gaussian process regression network (GPRN).

$$
K_{\mathrm{SM}-\mathrm{LMC}}=\sum_{q=1}^{Q} B^{q} \otimes K_{\mathrm{SM}}^{q}
$$

The $B^{q}$ in $K_{\mathrm{SM}-\mathrm{LMC}}$ encodes cross weights to represent task correlations and involves a linear combination of spectral mixtures. The CSM kernel [21] improved the expressiveness of SM-LMC: it 
contains cross phase spectrum and is also defined within the LMC framework as

$$
K_{\mathrm{CSM}}=\sum_{q=1}^{Q} B^{q} k_{S G}\left(\tau ; \Theta^{q}\right)
$$

where $k_{S G}\left(\tau ; \Theta^{q}\right)$ is phasor notation of the spectral Gaussian kernel. The kernels $k_{S G}\left(\tau ; \Theta^{q}\right)$ used in the CSM are, however, only phase dependent. The MOSM kernel [22] provides a principled framework to construct multivariate covariance functions with a better interpretation of cross relationships between tasks. MOSM has the form

$$
k_{\text {MOSM }}^{i j}(\tau)=\sum_{q=1}^{Q} \alpha_{i j}^{q} \exp \left(-\frac{1}{2}\left(\tau+\boldsymbol{\theta}_{i j}^{q}\right)^{\top} \Sigma_{i j}^{q}\left(\tau+\boldsymbol{\theta}_{i j}^{q}\right)\right) \cos \left(\left(\tau+\boldsymbol{\theta}_{i j}^{q}\right)^{\top} \boldsymbol{\mu}_{i j}^{q}+\phi_{i j}^{q}\right)
$$

where $\alpha_{i j}^{q}, \boldsymbol{\theta}_{i j}^{q}, \Sigma_{i j}^{q}, \boldsymbol{\mu}_{i j}^{q}$, and $\phi_{i j}^{q}$ are cross weight, cross time delay, cross covariance, cross mean, and cross phase delay between the $i$-th and $j$-th channels. SM-LMC and CSM are instances of MOSM. Even if MOSM improves upon existing methods in expressivity and interpretation, it still considers linear combinations of components and it ignores dependencies between spectral components.

A more recent SM based kernel for MTGP employs generalized convolution spectral mixture of coupling coregionalization (GCSM-CC) [23]. The GCSM-CC kernel is:

$$
K_{\mathrm{GCSM}-\mathrm{CC}}(\tau)=\sum_{i=1}^{Q} \sum_{j=1}^{Q} C_{i} C_{j}^{\top} \otimes K_{\mathrm{GCSM} i \times \mathrm{GCSM} j}
$$

where $C_{i} C_{j}^{\top}$ term is the coupling coregionalization term and $K_{\mathrm{GCSM} i \times \mathrm{GCSM} j}$ is the single task GCSM kernel. Although GCSM-CC considers both task level correlatons and spectral mixture level dependencies. GCSM-CC using coupling coregionalization, however, has the limitation that it shares the same kernel among tasks, which is a common drawback of MTGPs involving coregionalization [3]. Table $\mathbf{W}$ summarizes the characteristics of these kernels in terms of parameters and degrees of freedom.

Table 1: Comparisons between MT-GCSM and others. All LMC and coupling coregionalization terms use free form parameterization [3]

\begin{tabular}{lll}
\hline Kernel & Parameters & Degrees of freedom \\
\hline SE-LMC & $\left\{B, \theta_{f}, \theta_{\ell}\right\}$ & $2+\left(M^{2}+M\right) / 2$ \\
Matérn-LMC & $\left\{B, \theta_{f}, \theta_{\ell}\right\}$ & $2+\left(M^{2}+M\right) / 2$ \\
SM-LMC & $\left\{B^{q}, \boldsymbol{\mu}_{q}, \Sigma_{q}\right\}_{q=1}^{Q}$ & $Q\left(2 P+1+\left(M^{2}+M\right) / 2\right)$ \\
CSM & $\left\{\sigma^{q}, \mu^{q},\left\{\boldsymbol{w}_{r}^{q}, \boldsymbol{\phi}_{r}^{q}, \phi_{r}^{1 q} \triangleq_{0}\right\}_{r=1}^{M}\right\}_{q=1}^{Q}$ & $2 Q+M(2 Q-1)$ \\
MOSM & $\left\{\left\{w_{m}^{q}, \boldsymbol{\mu}_{m}^{q}, \Sigma_{m}^{q}, \boldsymbol{\theta}_{m}^{q}, \phi_{m}^{q}\right\}_{m=1}^{M}\right\}_{q=1}^{Q}$ & $Q M(3 P+2)$ \\
GCSM-CC & $\left\{B_{q}, w_{q}, \boldsymbol{\mu}_{q}, \Sigma_{q}, \boldsymbol{\theta}_{q}, \boldsymbol{\phi}_{q}\right\}_{q=1}^{Q}$ & $Q\left(4 P+1+\left(M^{2}+M\right) / 2\right)$ \\
MT-GCSM & $\left\{\left\{w_{q}^{m}, \boldsymbol{\mu}_{q}^{m}, \Sigma_{q}^{m}, \boldsymbol{\theta}_{q}^{m}, \boldsymbol{\phi}_{q}^{m}\right\}_{q=1}^{Q^{(m)}}\right\}_{m=1}^{M}$ & $\sum_{m=1}^{M} Q^{(m)}(4 P+1)$ \\
\hline
\end{tabular}

\section{Interpretation of the dependencies modeled in MT-GCSM}

MT-GCSM combines the advantages of MOSM and GCSM-CC without the above mentioned constraints, and is therefore more flexible and expressive. In this section we illustrate the dependencies modeled in MT-GCSM.

Figure B shows four outer cross convolution structures of two tasks in the time domain and its corresponding spectral densities in the frequency domain. Here each task have $Q=2$ base spectral mixtures. According to Equation (113), there are in total eight convolution structures (four inner full cross convolution structures plus four outer full cross convolution structures). Here we mainly 


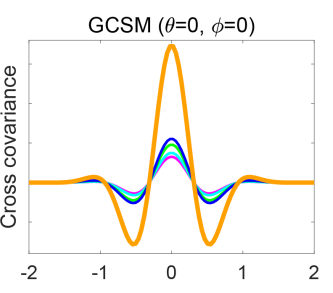

(a)

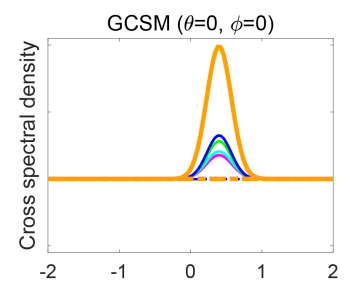

(e)

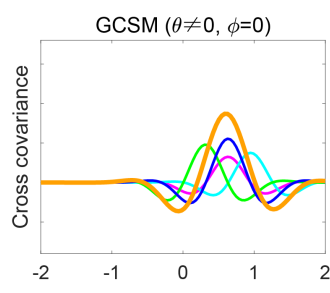

(b)

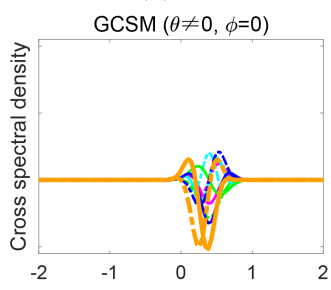

(f)

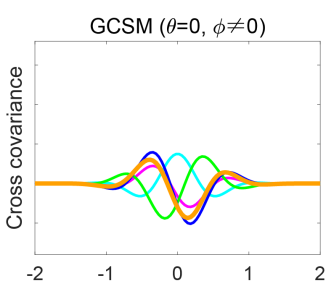

(c)

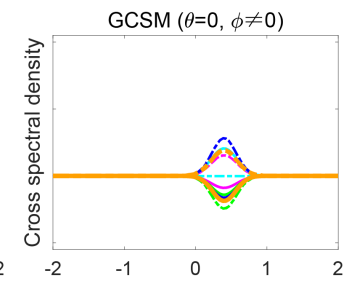

(g)

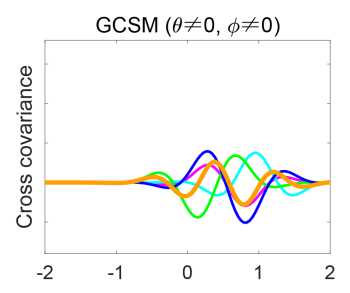

(d)

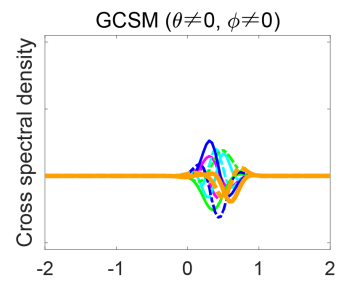

(h)

Figure 3: Cross covariance and corresponding spectral densities between tasks in $k_{\mathrm{MT}-\mathrm{GCSM}}^{\mathrm{T}_{m} \times \mathrm{T}_{m^{\prime}}}(\tau)$. The first row are zero time and phase delay, non-zero time delay and zero phase delay, zero time delay and non-zero phase delay, non-zero time and phase delay outer cross convolution structures of task $T_{1}\left(Q^{(1)}=2\right)$ and task $T_{2}\left(Q^{(2)}=2\right)$ in MT-GCSM. The orange bolder solid lines are sum of outer cross convolution structures in MT-GCSM. The second row are corresponding spectral densities of the first row. For spectral densities of outer cross convolution structures, real part in solid line and imaginary part in dashed line.

give the four outer full cross convolution structures (see Equation ([13)) because the four inner full convolution structures are given by single task GCSM (see Equation (III) [24]). The first row in Figure 3 show four full cross convolution structures (solid line with different color) in MT-GCSM and its sum respectively. From the last row in Figure 4, We can see the corresponding cross spectral densities in the frequency domain. Analyzing Equation [13 demonstrates that closer frequencies $\left(\boldsymbol{\mu}_{i}^{m}, \boldsymbol{\mu}_{j}^{m^{\prime}}\right)$, scales $\left(\Sigma_{i}^{m}, \Sigma_{j}^{m^{\prime}}\right)$, and weights $\left(w_{i}^{m}, w_{j}^{m^{\prime}}\right)$ between tasks $T_{m}$ and $T_{m^{\prime}}$ in MT-GCSM, induce stronger cross dependencies.

\section{Experiments}

In general, SM based kernels are sensitive to the initialization of its hyper-parameters, which can lead to a local optimal solution for NLML (see Equation 6). MT-GCSM kernel has the same initialization problem. In MT-GCSM, the degrees of freedom and parameter space are $M$ times as large compared to using GCSM with a single task. Hyper-parameters initialization has a direct impact on the ability to discover and extrapolate patterns, especially in the presence of complex multiple tasks. Therefore we apply an initialization strategy which uses the empirical spectral density, which has been shown to be effective in other contexts [25]. The empirical spectral density is, however, often noisy, so its direct use is not possible. Past research suggests that sharp peaks of the empirical spectral density are near the true frequencies [25, 34]. We make use of this observation, and apply a Bayesian Gaussian mixture model $p(\boldsymbol{\theta} \mid \boldsymbol{s})=\sum_{i=1}^{Q} \tilde{w}_{i} \mathcal{N}\left(\tilde{\boldsymbol{\mu}}_{i}, \tilde{\Sigma}_{i}\right)$ on the empirical spectral density $\mathbf{s}$ in order to get the $Q$ cluster centers of the Gaussian spectral density. We use the Expectation Maximization algorithm [35] to estimate the parameters $\tilde{w}_{i}, \tilde{\boldsymbol{\mu}}_{i}$, and $\tilde{\Sigma}_{i}$. The results are used as initial values of $w_{i}, \boldsymbol{\mu}_{i}$, and $\Sigma_{i}$ in each task, respectively. Then the time and phase delay parameters $\left\{\boldsymbol{\theta}_{i}, \boldsymbol{\phi}_{i}\right\}$ are randomly initialized for each task in MT-GCSM. We use this technique in all our experiments on artificial and real world data.

We compare MT-GCSM with published MTGP methods, namely SM-LMC, CSM, MOSM, and GCSM-CC. The same initialization strategy used for MT-GCSM was also used in SM-LMC, CSM, MOSM, and GCSM-CC. The coregionalization terms in GCSM-CC are randomly initialized. In order to show the stronger structure learning ability of the proposed MT-GCSM, with considering tasks should have different complexity, we set MT-GCSM to use less number of components in each 
task for all experiments. First we show the ability of MT-GCSM to model a mixed signal sampled from a Gaussian distribution specified by $\mathcal{G P}\left(0, K_{\mathrm{SM}}(Q=3)\right)$, its integral and derivative, and its time delayed signal simultaneously. Then we use MT-GCSM for prediction tasks on a real-world problem with two sensor array datasets: humidity monitoring related to climate change, Nitrogen dioxide $\left(\mathrm{NO}_{2}\right)$ concentration related to air pollution. We implemented our models in Tensorflow [36] and GPflow [37] to improve scalability and to facilitate gradient computation. In all experiments we use the mean absolute error MAE $=\sum_{i=1}^{n}\left|y_{i}-\tilde{y}_{i}\right| / n$ as performance metric.

\subsection{Learning Signal, integral, derivative and time delayed signal simultaneously}

We design an artificial experiment in order to validate the interpolation, extrapolation, signal recovery, and block miss filling ability of MT-GCSM and compare its structure learning performance with that of other MTGP methods. We generate four nonlinear correlated tasks: a mixed signal, its integral, its derivative, and its time delay, respectively. Specifically, we sample a Gaussian signal $f(x) \sim \mathcal{G} \mathcal{P}\left(0, K_{\mathrm{SM}}(Q=3)\right)$ with length 300 in the interval $[-10,10]$, numerically compute its first integral and derivative, and delay the signal with $f(x)=f(x+t),(t=2)$.

From the signal $f(x)$ we randomly choose half of the data as training data, and the rest as test data. The integration signal points in the interval $[-10,0]$ are used for training (in cyan), while the remaining signal points in the interval $[0,10]$ are used for testing (in green). For the third task, the derivative of the signal, data in the interval $[0,10]$ is used for training and the rest is used for testing. In the fourth task, signal points in the intervals $[7,10]$ and $[-8,-3]$ are used for training and the rest of the points in the middle interval $[-3,7]$ are used for testing.

The performance of MT-GCSM on the generated signal is shown in Figure 4a. As shown in Table ฉ, all considered GP methods have comparable performance: they learn the covariance between tasks and are able to interpolate missing values well. The second task, i.e., the integral of the signal, is shown in Figure 4b. In this case its inherent patterns are more difficult to recognize and extrapolate. Here MT-GCSM and GCSM-CC perform better than other methods, but MT-GCSM achieves lowest MAE as well as smallest confidence interval. Both MT-GCSM and GCSM-CC excel also on the last two tasks. For instance, the derivative signal (Figure 4d) and the time delay signal (Figure 4d): here MT-GCSM and GCSM-CC show stronger pattern learning and extrapolation capability, with MT-GCSM having the best performance. Predictions obtained using SE-LMC and Matérn-LMC kernels are of low quality, especially for the long range extrapolation tasks (integral, derivative, and time delay signals): it is very hard for them to find valid patterns in the data, like the change of trend over time. Overall, results indicate the capability of MT-GCSM to capture integration and differentiation patterns of the generated signal simultaneously. Note that here SMLMC, CSM, MOSM, GCSM-CC have the same $Q=10$ with 10, 10, 10, and 100 components, respectively, but MT-GCSM has a different number of base spectral mixtures for each $\operatorname{task}\left(Q^{(1)}=4\right.$, $Q^{(2)}=5, Q^{(3)}=6, Q^{(4)}=5$ ). In total, we have $\sum_{i=1}^{4} \sum_{j=1}^{4} Q^{(i)} Q^{(j)}=400$ convolution structures (102 inner convolution structures and 298 outer convolution structures) in MT-GCSM, which can capture far more structures in the data without the need of using extra hyper-parameters. As result of applying full cross convolution and considering complexity diversity of tasks, MTGCSM using less number of components can learn patterns and dependencies better than the other kernels. Table $\square$ shows that only MT-GCSM gives a MAE smaller than 0.1 for all tasks, indicating the advantage of MT-GCSM compared to other kernels.

Table 2: Performance comparisons between MT-GCSM and others kernels on artificial dataset. The MT-GCSM kernel consistently presents the lowest MAE.

\begin{tabular}{cllll}
\hline Kernel & $\mathcal{G P}\left(0, K_{\mathrm{SM}}\right)$ & Integral & Derivative & Time delay \\
\hline SE-LMC & 0.157 & 0.184 & 0.202 & 0.809 \\
Matérn-LMC & 0.128 & 0.168 & 0.198 & 0.642 \\
SM-LMC & 0.123 & 0.198 & 0.192 & 0.503 \\
CSM & 0.130 & 0.170 & 0.202 & 0.603 \\
MOSM & 0.137 & 0.163 & 0.203 & 0.535 \\
GCSM-CC & 0.132 & 0.094 & 0.127 & 0.327 \\
MT-GCSM & $\mathbf{0 . 0 8 7}$ & $\mathbf{0 . 0 5 9}$ & $\mathbf{0 . 0 4 4}$ & $\mathbf{0 . 0 9 6}$ \\
\hline
\end{tabular}



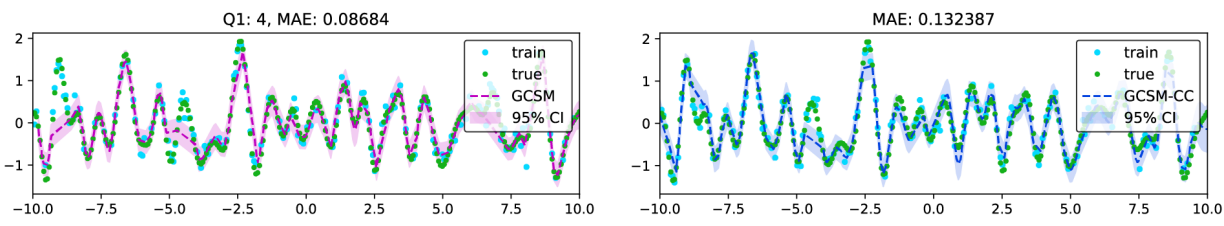

(a) Signal $\sim \mathcal{G P}\left(0, K_{\mathrm{SM}}(Q=3)\right)$
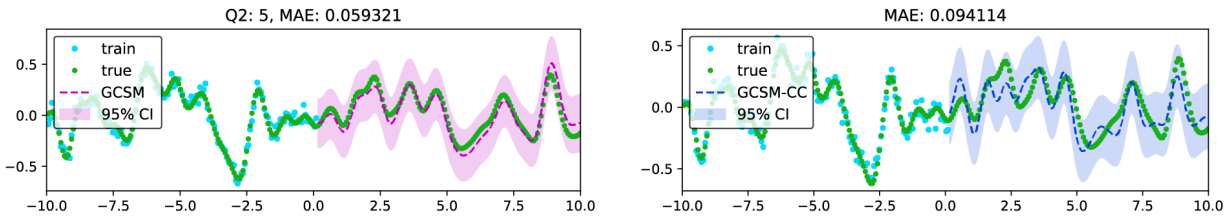

(b) Integral of the signal
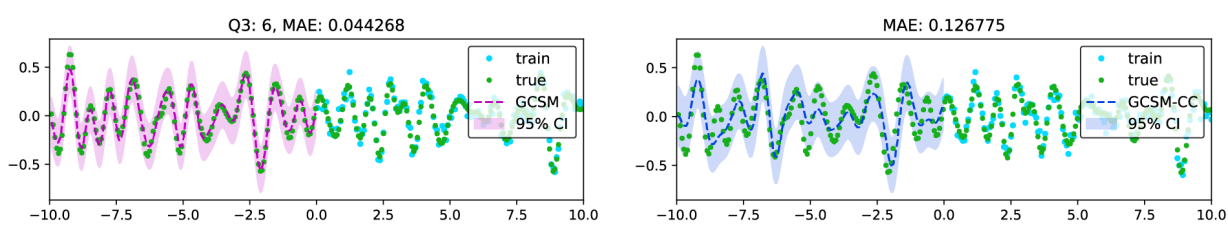

(c) Derivative of the signal
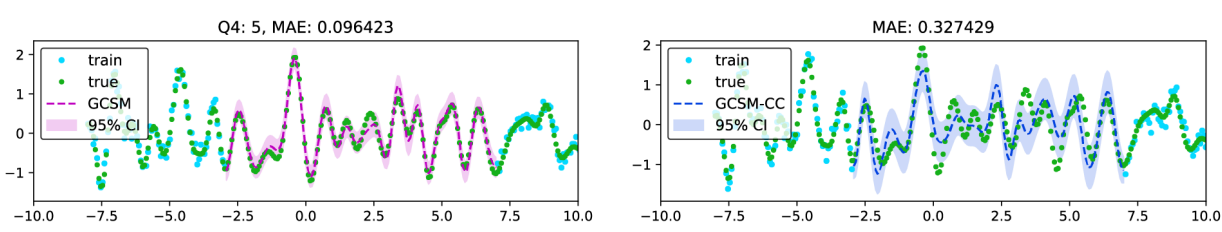

(d) Task level time delayed signal

Figure 4: Performance of MT-GCSM (in dashed magenta line) and GCSM-CC (in dashed blue line) on artificial dataset. The shaded area shows the predicted variance. In this case only MT-GCSM can correctly learning the structure of four tasks simultaneously.

\subsection{Humidity long range extrapolation}

Sensor networks monitoring climate change in Stockholm city ${ }^{\text {m }}$ provide historical analysis and information about the future evolution of the regional environment. The recording allows us a possibility to extrapolate long range meteorological parameters such as humidity, which could guide environmental policy making. On the other hand, meteorological parameters are one of the main factors affecting local air pollution because they determine how air pollutant spreads. The humidity monitoring recordings are recorded from a number of stations (Torkel Knutssonsgatan, Marsta, Norr Malma, Högdalen) in Stockholm and outside. For instance: Torkel Knutssonsgatan's measurement at urban background, Marsta's measurement and Högdalen's measurement at a high-altitude tower, North Malma's measurement at the regional background. Stockholm as a seaside city, the change of humidity in the city not only depends on weather conditions but also depends on its geographical location and surroundings. For example, stations located in the same area definitely have the same weather condition but will have totally different humidity values, because stations located nearby river or seaside will have higher humidity values. These factors are time and phase dependent with different scales.

\footnotetext{
${ }^{1}$ http://slb.nu/slbanalys/historiska-data-luft/
} 
In this case, we use humidity time series from 05 November 2017 to 25 November 2017, in 1 hour intervals. Considering the advantage of structure learning and complexity diversity in MT-GCSM, here we set less number of components $Q^{(1)}=5, Q^{(2)}=6, Q^{(3)}=7$, and $Q^{(4)}=5$ for task 1 , task 2, task 3, and task 4, respectively. Here, randomly chosen half of humidity data in Torkel Knutssonsgatan (task 1), the first half of humidity data in Marsta (task 2), the last half of humidity data in Norr Malma (task 3), the first quarter and last quarter of humidity data in Högdalen (task 4) are used for training, the last half of humidity data in Marsta and the middle part of humidity data in Högdalen are used for testing. We aim to extrapolate the long range missing values of humidity data in Marsta and middle block missing values of humidity data in Högdalen. From Figure 5, we can see that there are no stable background environment values in the humidity recording. Interestingly, over time high peaks in humidity are more stable than low peaks. From Figure [5, in addition to time and phase dependent patterns within tasks (local patterns depending on surroundings) and time and phase dependent patterns cross tasks (global patterns depending on seasonal or yearly factors), we observe that the low peaks in humidity appear irregularly. The change in humidity is complicated and caused by the nonlinear interaction of local and global patterns. Therefore data from multiple stations should help when used to model long range extrapolation trends. Results indicate that all SM based kernels can extrapolate the humidity well, with MT-GCSM consistently achieving better performance in terms of MAE and predicted confidence interval (see Figure 5 and Table B]).

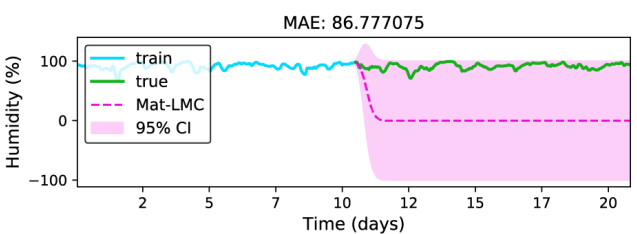

(a) Matérn-LMC

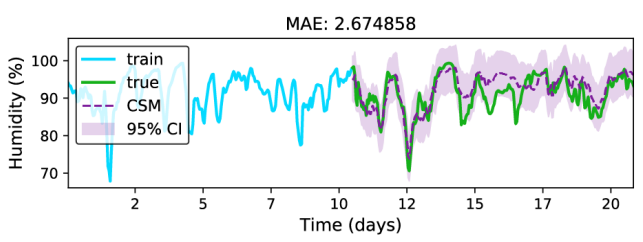

(c) CSM

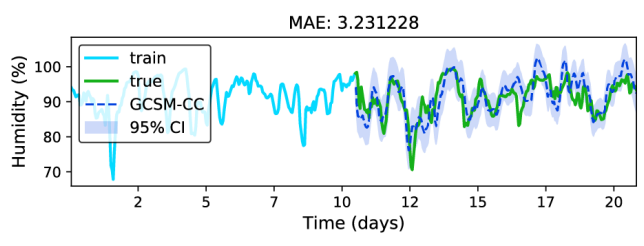

(e) GCSM-CC

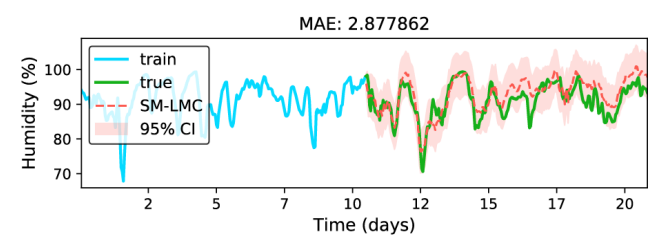

(b) SM-LMC

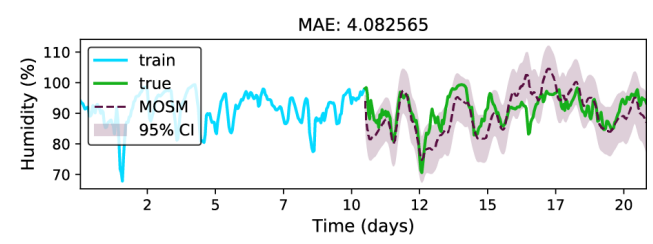

(d) MOSM

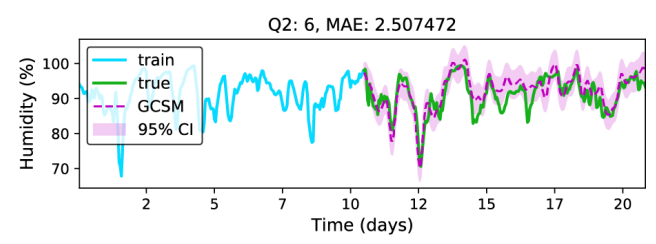

(f) MT-GCSM

Figure 5: Performance comparison between MT-GCSM and other kernels on long range humidity extrapolation: (a) Matérn-LMC (in pink dashed line), (b) SM-LMC (in red dashed line), (c) CSM (in purple dashed line), (d) MOSM (in plum dashed line), (e) GCSM-CC (in blue dashed line), (f) MT-GCSM (in dashed magenta line) configured $Q=\{5,6,7,5\}$ for tasks. The shaded area shows the predicted variance.

\subsection{Nitrogen dioxide concentration long range extrapolation}

As second real world experiment we use $\mathrm{NO}_{2}$ concentration recordings in sensor networks. The Nitrogen dioxides $\left(\mathrm{NO}_{2}\right)$ is an important air pollution parameter reflecting the increase of industry fossil fuels emission and automobile exhaust, which causes inflammation of the airways if humans suffer from long term exposure at a high concentration. If we can discover the variation in $\mathrm{NO}_{2}$ concentration, using pattern recognition, this may suggest how to control and prevent the negative effects on our health and the environment. In this case, the $\mathrm{NO}_{2}$ concentration recordings are collected from four stations in Stockholm city: Essingeleden's measurement at open path, Hornsgatan's, 
Sveavägen's, and Norrlandsgatan's measurement at a street. All recordings cover 24 hours at 1 hour interval and missing values are filtered. Each station corresponds to a task: Essingeleden as task 1, Hornsgatan as task 2, Sveavägen as task 3, and Norrlandsgatan as task 4. $\mathrm{NO}_{2}$ evolution has time and phase related patterns. Different stations have different local patterns which depend on the station's surroundings. Still, these tasks have shared global trends because of the global seasonal change and periodic characteristics of human and industry activities. The evolution of $\mathrm{NO}_{2}$ concentration in each task is a result of nonlinear interaction of time and phase dependent local and global patterns.

In this case, we consider the recording time for $\mathrm{NO}_{2}$ concentration from $25 \mathrm{July}, 2017$ to 15 August, 2017. From Figure 6, in addition to time and phase dependent global trends (global patterns depending on seasonal or yearly factors) cross tasks and local patterns (local patterns depending on local human activities and surrounding) within task, we observe that the high peaks in $\mathrm{NO}_{2}$ appear nonperiodically. Here we randomly choose half of $\mathrm{NO}_{2}$ data in Essingeleden, the first half of $\mathrm{NO}_{2}$ data in Hornsgatan, the last half of $\mathrm{NO}_{2}$ data in Sveavägen, and the first quarter and last quarter of $\mathrm{NO}_{2}$ data in Norrlandsgatan for training. The last half of $\mathrm{NO}_{2}$ data in Hornsgatan and the middle part of $\mathrm{NO}_{2}$ data in Norrlandsgatan are used for testing. We aim to extrapolate the long range missing values of $\mathrm{NO}_{2}$ data in Hornsgatan and middle block missing values of $\mathrm{NO}_{2}$ data in Norrlandsgatan. In this case, we aiming to give learning superiority of MT-GCSM even with less number of components for each task. Taking complexity diversity of tasks in account, we use $Q=\{4,5,5,4\}$ for MT-GCSM and $Q=10$ for the other kernels. Results indicate that all SM based kernels can extrapolate the future $\mathrm{NO}_{2}$ concentration well, with MT-GCSM achieving the best performance (see Figure 6 and Table 3 ) in task 3 and GCSM-CC achieving the best performance in task 4 . In this experiment, GCSM based kernels perform better than other kernels, which means there are indeed dependent structure in the $\mathrm{NO}_{2}$ concentration data. Actually predictions using the SE-LMC and Matérn-LMC cannot discover any valid patterns (see Figure 6a).

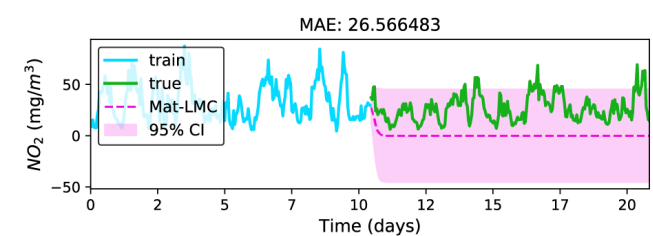

(a) Matérn-LMC

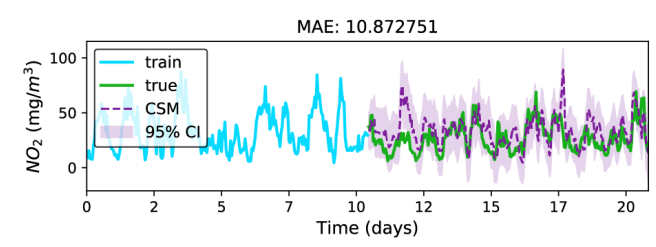

(c) CSM

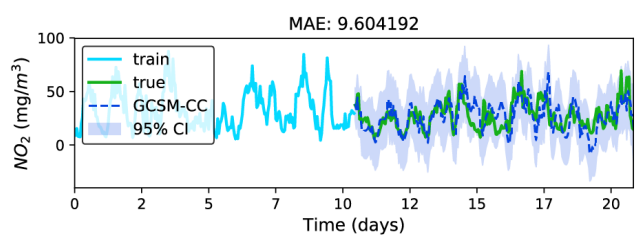

(e) GCSM-CC

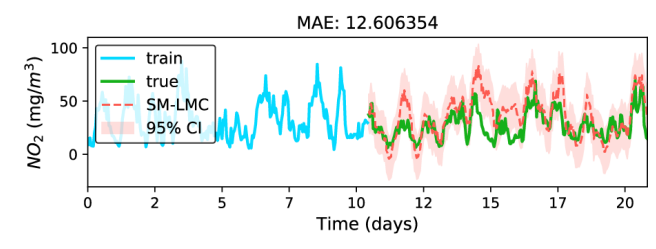

(b) SM-LMC

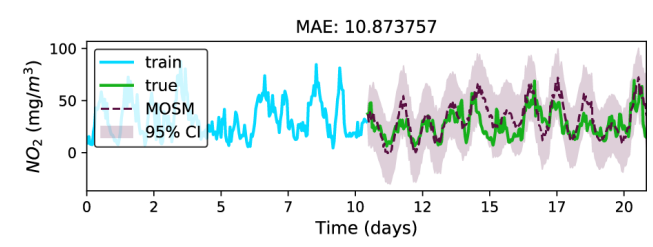

(d) MOSM

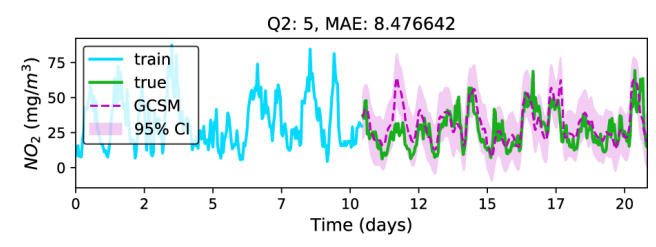

(f) CGCSM

Figure 6: Performance comparison between MT-GCSM and other kernels on long range $\mathrm{NO}_{2}$ extrapolation: (a) Matérn-LMC (in pink dashed line), (b) SM-LMC (in red dashed line), (c) CSM (in purple dashed line), (d) MOSM (in plum dashed line), (e) GCSM-CC (in blue dashed line), (f) MTGCSM (in dashed magenta line) configured $Q=\{4,5,5,4\}$ for tasks. The shaded area shows the predicted variance. 
Table 3: Performance comparison between MT-GCSM and other kernels on real world datasets. The superscript $\mathrm{M}$ and $\mathrm{H}$ in humidity are Marsta station and Högdalen station, respectively. The superscript $\mathrm{H}$ and $\mathrm{N}$ in $\mathrm{NO}_{2}$ are Hornsgatan station and Norrlandsgatan station, respectively

\begin{tabular}{ccccc}
\hline Kernel & Humidity $^{M}$ & Humidity $^{H}$ & $\mathrm{NO}_{2}^{H}$ & $\mathrm{NO}_{2}^{N}$ \\
\hline SE-LMC & 77.267 & 63.456 & 26.010 & 26.607 \\
Matérn-LMC & 86.777 & 80.493 & 26.566 & 27.288 \\
SM-LMC & 2.878 & 2.066 & 12.606 & 8.803 \\
CSM & 2.675 & 3.043 & 10.873 & 9.260 \\
MOSM & 4.083 & 2.929 & 10.874 & 8.632 \\
GCSM-CC & 3.231 & 2.068 & 9.604 & $\mathbf{7 . 7 9 6}$ \\
MT-GCSM & $\mathbf{2 . 5 0 7}$ & $\mathbf{2 . 0 0 9}$ & $\mathbf{8 . 4 7 7}$ & 8.175 \\
\hline
\end{tabular}

\section{Discussion}

We proposed the multi-task generalized convolution spectral mixture (MT-GCSM) kernel to model dependent structures in tasks and across tasks. MT-GCSM is able to extrapolate multiple complex tasks simultaneously by using inner and cross convolution of time and phase dependent spectral mixtures. Experiments on artificial and real world datasets have shown the superiority that the MTGCSM with less number of components for each task, is capable to exploit task level correlation and spectral mixture level dependency at the spectral mixture scale in the frequency domain. When used in multitask GPs, MT-GCSM can recognize and model complex structure in data, discover nonlinear correlation between tasks, and can make long-term extrapolations. Notably, MT-GCSM allows tasks to have a different number of components, which is more natural, as each task cannot be guaranteed to have the same number of patterns.

The proposed MT-GCSM kernel is an extension of previous work to discover spectral mixture level dependency within a task as well as task level nonlinear correlation between tasks. A limitation of the proposed kernel, shared by MTGPs which use multiple kernels, is the resulting relative inefficient inference. Sparse approximation and Bayesian optimization can be adopted as an improvement in efficient inference and hyper-parameters initialization [32, 38, 39, 40], which should improve the scalability of MT-GCSM. Currently efficient inference approximation methods like FITC and PITC [15, [16, 41, 42, 43], are not very effective for MTGPs using a multi-kernel framework. Future research will focus on sparse representations or efficient inference of the MT-GCSM.

\section{Acknowledgement}

This work was partly supported by China Scholarship Council (CSC).

\section{References}

[1] C. E. Rasmussen and H. Nickisch, "Gaussian processes for machine learning (gpml) toolbox," Journal of Machine Learning Research, vol. 11, no. Nov, pp. 3011-3015, 2010.

[2] C. E. Rasmussen, Gaussian processes for machine learning, ser. Adaptive computation and machine learning, C. K. I. Williams, Ed. Cambridge, Massachusetts: The MIT Press, 2006.

[3] E. V. Bonilla, K. M. Chai, and C. Williams, "Multi-task Gaussian process prediction," in $\mathrm{Ad}$ vances in neural information processing systems, 2008, pp. 153-160.

[4] Z. Xu and K. Kersting, "Multi-task learning with task relations," in Proc. IEEE 11th Int. Conf. Data Mining, Dec. 2011, pp. 884-893.

[5] H. Kang and S. Choi, "Bayesian multi-task learning for common spatial patterns," in Proc. Int. Workshop Pattern Recognition in NeuroImaging, May 2011, pp. 61-64.

[6] G. Leen, J. Peltonen, and S. Kaski, "Focused multi-task learning in a Gaussian process framework," Machine Learning, vol. 89, no. 1-2, pp. 157-182, 2012. 
[7] R. Dürichen, M. A. Pimentel, L. Clifton, A. Schweikard, and D. A. Clifton, "Multitask Gaussian processes for multivariate physiological time-series analysis," IEEE Transactions on Biomedical Engineering, vol. 62, no. 1, pp. 314-322, 2015.

[8] W. Ruan, A. B. Milstein, W. Blackwell, and E. L. Miller, "Multiple output Gaussian process regression algorithm for multi-frequency scattered data interpolation," in Proc. IEEE Int. Geoscience and Remote Sensing Symp. (IGARSS), Jul. 2017, pp. 3992-3995.

[9] T. V. Nguyen and E. V. Bonilla, "Collaborative multi-output Gaussian processes," in Proceedings of the Thirtieth Conference on Uncertainty in Artificial Intelligence, UAI 2014, Quebec City, Quebec, Canada, July 23-27, 2014, N. L. Zhang and J. Tian, Eds. AUAI Press, 2014, pp. 643-652. [Online]. Available: https://dslpitt.org/uai/displayArticleDetails. jsp?mmnu=1\&smnu=2\&article_id=2500\&proceeding_id=30

[10] H. Wackernagel, "Multivariate geostatistics, 387 pp," 2003.

[11] A. Melkumyan and F. Ramos, "Multi-kernel Gaussian processes," in IJCAI ProceedingsInternational Joint Conference on Artificial Intelligence, vol. 22, no. 1, 2011, p. 1408.

[12] I. Bilionis and N. Zabaras, "Multi-output local Gaussian process regression: applications to uncertainty quantification," Journal of Computational Physics, vol. 231, no. 17, pp. 57185746, 2012.

[13] B. Zhang, B. A. Konomi, H. Sang, G. Karagiannis, and G. Lin, "Full scale multi-output Gaussian process emulator with nonseparable auto-covariance functions," Journal of Computational Physics, vol. 300, pp. 623-642, 2015.

[14] P. Goovaerts, "Geostatistics for natural resources evaluation. oxford univ. press, new york." Geostatistics for natural resources evaluation. Oxford Univ. Press, New York., 1997.

[15] M. Alvarez and N. D. Lawrence, "Sparse convolved Gaussian processes for multi-output regression,” in Advances in neural information processing systems, 2009, pp. 57-64.

[16] M. A. Álvarez and N. D. Lawrence, "Computationally efficient convolved multiple output Gaussian processes," Journal of Machine Learning Research, vol. 12, no. May, pp. 1459-1500, 2011.

[17] S. Gómez-González, M. A. Álvarez, H. F. García, J. I. Ríos, and A. A. Orozco, "Discriminative training for convolved multiple-output Gaussian processes," in Iberoamerican Congress on Pattern Recognition. Springer, 2015, pp. 595-602.

[18] C. Guarnizo and M. A. Álvarez, "Fast kernel approximations for latent force models and convolved multiple-output Gaussian processes," arXiv preprint arXiv:1805.07460, 2018.

[19] A. G. Wilson, "Covariance kernels for fast automatic pattern discovery and extrapolation with Gaussian processes," University of Cambridge, 2014.

[20] A. G. Wilson, D. A. Knowles, and Z. Ghahramani, "Gaussian process regression networks," arXiv preprint arXiv:1110.4411, 2011.

[21] K. R. Ulrich, D. E. Carlson, K. Dzirasa, and L. Carin, "GP kernels for cross-spectrum analysis," in Advances in neural information processing systems, 2015, pp. 1999-2007.

[22] G. Parra and F. Tobar, "Spectral mixture kernels for multi-output Gaussian processes," in $A d$ vances in Neural Information Processing Systems, 2017, pp. 6684-6693.

[23] K. Chen, P. Groot, J. Chen, and E. Marchiori, "Generalized Spectral Mixture Kernels for MultiTask Gaussian Processes,” ArXiv e-prints, Aug. 2018.

[24] _ , "Spectral Mixture Kernels with Time and Phase Delay Dependencies," ArXiv e-prints, Aug. 2018.

[25] A. Wilson and R. Adams, "Gaussian process kernels for pattern discovery and extrapolation," in Proceedings of the 30th International Conference on Machine Learning (ICML-13), 2013, pp. 1067-1075.

[26] S. Bochner, Lectures on Fourier Integrals.(AM-42). Princeton University Press, 2016, vol. 42.

[27] M. Stein, "Interpolation of spatial data: some theory for kriging. 1999."

[28] A. G. Wilson, E. Gilboa, A. Nehorai, and J. P. Cunningham, "Fast kernel learning for multidimensional pattern extrapolation," in Advances in Neural Information Processing Systems, 2014, pp. 3626-3634. 
[29] D. Duvenaud, J. R. Lloyd, R. Grosse, J. B. Tenenbaum, and Z. Ghahramani, "Structure discovery in nonparametric regression through compositional kernel search," arXiv preprint arXiv:1302.4922, 2013.

[30] S. Flaxman, A. Wilson, D. Neill, H. Nickisch, and A. Smola, "Fast kronecker inference in Gaussian processes with non-gaussian likelihoods," in International Conference on Machine Learning, 2015, pp. 607-616.

[31] J. B. Oliva, A. Dubey, A. G. Wilson, B. Póczos, J. Schneider, and E. P. Xing, "Bayesian nonparametric kernel-learning," in Artificial Intelligence and Statistics, 2016, pp. 1078-1086.

[32] P. A. Jang, A. Loeb, M. Davidow, and A. G. Wilson, "Scalable Levy process priors for spectral kernel learning," in Advances in Neural Information Processing Systems, 2017, pp. 3943-3952.

[33] S. Remes, M. Heinonen, and S. Kaski, "A mutually-dependent hadamard kernel for modelling latent variable couplings," in Asian Conference on Machine Learning, 2017, pp. 455-470.

[34] W. Herlands, A. Wilson, H. Nickisch, S. Flaxman, D. Neill, W. Van Panhuis, and E. Xing, "Scalable gaussian processes for characterizing multidimensional change surfaces," in Artificial Intelligence and Statistics, 2016, pp. 1013-1021.

[35] T. K. Moon, "The expectation-maximization algorithm," IEEE Signal Processing Magazine, vol. 13, no. 6, pp. 47-60, 1997.

[36] M. Abadi, P. Barham, J. Chen, Z. Chen, A. Davis, J. Dean, M. Devin, S. Ghemawat, G. Irving, M. Isard et al., "Tensorflow: A system for large-scale machine learning. arxiv preprint," arXiv preprint arXiv:1605.08695, 2016.

[37] A. G. d. G. Matthews, M. van der Wilk, T. Nickson, K. Fujii, A. Boukouvalas, P. León-Villagrá, Z. Ghahramani, and J. Hensman, "GPflow: A Gaussian process library using tensorflow,” Journal of Machine Learning Research, vol. 18, no. 40, pp. 1-6, 2017.

[38] K. Swersky, J. Snoek, and R. P. Adams, "Multi-task Bayesian optimization," in Advances in neural information processing systems, 2013, pp. 2004-2012.

[39] R. Martinez-Cantin, "Bayesopt: A Bayesian optimization library for nonlinear optimization, experimental design and bandits," The Journal of Machine Learning Research, vol. 15, no. 1, pp. 3735-3739, 2014.

[40] N. Knudde, J. van der Herten, T. Dhaene, and I. Couckuyt, "GPflowopt: A Bayesian optimization library using tensorflow," arXiv preprint arXiv:1711.03845, 2017.

[41] Y. Wang and R. Khardon, "Sparse Gaussian processes for multi-task learning," in Joint European Conference on Machine Learning and Knowledge Discovery in Databases. Springer, 2012, pp. 711-727.

[42] J. Quiñonero-Candela and C. E. Rasmussen, "A unifying view of sparse approximate Gaussian process regression," Journal of Machine Learning Research, vol. 6, no. Dec, pp. 1939-1959, 2005.

[43] K. Chalupka, C. K. Williams, and I. Murray, "A framework for evaluating approximation methods for Gaussian process regression,” Journal of Machine Learning Research, vol. 14, no. Feb, pp. 333-350, 2013. 


\section{Supplementary}

\section{A Positive semi-definite condition of MT-GCSM}

The proof of positive semi-definite condition of MT-GCSM is different from single task GCSM because of the introduction of outer cross convolution terms between tasks. In MT-GCSM, given samples $\left\{x_{1}^{(1)}, x_{2}^{(1)}, \ldots, x_{n}^{(1)}\right\} \in \operatorname{task}^{(1)}$ and $\left\{x_{1}^{(2)}, x_{2}^{(2)}, \ldots, x_{m}^{(2)}\right\} \in \operatorname{task}^{(2)}$, if there are arbitrary numbers $\left\{a_{1}^{(1)}, a_{2}^{(1)}, \ldots, a_{n}^{(1)}\right\}$ and $\left\{a_{1}^{(2)}, a_{2}^{(2)}, \ldots, a_{m}^{(2)}\right\}$, we have a quadratic form

$$
\begin{aligned}
K_{\text {MT-GCSM }}\left(x^{(1)}, x^{(2)}\right) & =\boldsymbol{\Omega} \\
& =\left(A, A^{\prime}\right)\left(\begin{array}{ll}
\Omega_{11} & \Omega_{12} \\
\Omega_{21} & \Omega_{22}
\end{array}\right)\left(\begin{array}{c}
A^{\top} \\
A^{\prime \top}
\end{array}\right)
\end{aligned}
$$

where

$$
\begin{aligned}
A & =\left\{a_{1}^{(1)}, a_{2}^{(1)}, \ldots, a_{n}^{(1)}\right\} \\
A^{\prime} & =\left\{a_{1}^{(2)}, a_{2}^{(2)}, \ldots, a_{m}^{(2)}\right\} \\
\Omega_{11} & =\left\{K^{1,1}\left(x_{i}^{(1)}, x_{j}^{(1)}\right)\right\}_{i, j=1}^{n} \\
\Omega_{22} & =\left\{K^{2,2}\left(x_{l}^{(2)}, x_{t}^{(2)}\right)\right\}_{l, t=1}^{m} \\
\Omega_{12} & =\left\{K^{1,2}\left(x_{i}^{(1)}, x_{t}^{(2)}\right)\right\}_{i=1 ; t=1}^{n ; m} \\
\Omega_{21} & =\left\{K^{2,1}\left(x_{l}^{(2)}, x_{j}^{(1)}\right)\right\}_{l=1 ; j=1}^{m ; n}
\end{aligned}
$$

According to Equation $\mathbb{}$, we have the integral form of $K_{\mathrm{GCSM}}$ in each task.

$$
\begin{aligned}
& K_{\mathrm{GCSM}}^{1,1}\left(x^{(1)}, x^{(1)^{\prime}}\right)=\int_{-\infty}^{+\infty} \sum_{q_{1}=1}^{Q^{(1)}} \sum_{q_{2}=1}^{Q^{(1)}} g_{q_{1}}^{(1)}\left(x^{(1)}-u\right) g_{q_{2}}^{(1)}\left(x^{(1)^{\prime}}-u\right) d u \\
& K_{\mathrm{GCSM}}^{2,2}\left(x^{(2)}, x^{(2)^{\prime}}\right)=\int_{-\infty}^{+\infty} \sum_{q_{1}=1}^{Q^{(2)}} \sum_{q_{2}=1}^{Q^{(2)}} g_{q_{1}}^{(2)}\left(x^{(2)}-u\right) g_{q_{2}}^{(2)}\left(x^{(2)^{\prime}}-u\right) d u \\
& K_{\mathrm{GCSM}}^{1,2}\left(x^{(1)}, x^{(2)}\right)=\int_{-\infty}^{+\infty} \sum_{q_{1}=1}^{Q^{(1)}} \sum_{q_{2}=1}^{Q^{(2)}} g_{q_{1}}^{(1)}\left(x^{(1)}-u\right) g_{q_{2}}^{(2)}\left(x^{(2)}-u\right) d u \\
& K_{\mathrm{GCSM}}^{2,1}\left(x^{(2)}, x^{(1)}\right)=\int_{-\infty}^{+\infty} \sum_{q_{1}=1}^{Q^{(2)}} \sum_{q_{2}=1}^{Q^{(1)}} g_{q_{1}}^{(2)}\left(x^{(1)}-u\right) g_{q_{2}}^{(1)}\left(x^{(2)}-u\right) d u
\end{aligned}
$$

Then

$$
\begin{aligned}
\boldsymbol{\Omega} & =A \Omega_{11} A^{\top}+A^{\prime} \Omega_{21} A^{\top}+A \Omega_{12} A^{\prime \top}+A^{\prime} \Omega_{22} A^{\prime^{\top}} \\
& =\int_{-\infty}^{+\infty}\left(\sum_{i=1}^{n} \sum_{q_{1}=1}^{Q^{(1)}} a_{i}^{(1)} g_{q_{1}}^{(1)}\left(x_{i}^{(1)}-u\right)+\sum_{l=1}^{m} \sum_{q_{2}=1}^{Q^{(2)}} a_{l}^{(2)} g_{q_{2}}^{(2)}\left(x_{l}^{(1)}-u\right)\right)^{2} \geq 0
\end{aligned}
$$

In above Equations (118) and (21), each task has free number of components. Arbitrary MTGP kernel $\Omega$ constructed by MT-GCSM with arbitrary number of tasks fulfills the positive semi-definite condition $(\boldsymbol{\Omega} \geq 0)$. 